Vesselin Drensky · Jie-Tai Yu

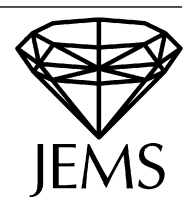

\title{
The Strong Anick Conjecture is true
}

Received November 1, 2005

\begin{abstract}
Recently Umirbaev has proved the long-standing Anick conjecture, that is, there exist wild automorphisms of the free associative algebra $K\langle x, y, z\rangle$ over a field $K$ of characteristic 0 . In particular, the well-known Anick automorphism is wild. In this article we obtain a stronger result (the Strong Anick Conjecture that implies the Anick Conjecture). Namely, we prove that there exist wild coordinates of $K\langle x, y, z\rangle$. In particular, the two nontrivial coordinates in the Anick automorphism are both wild. We establish a similar result for several large classes of automorphisms of $K\langle x, y, z\rangle$. We also find a large new class of wild automorphisms of $K\langle x, y, z\rangle$ which is not covered by the results of Umirbaev. Finally, we study the lifting problem for automorphisms and coordinates of polynomial algebras, free metabelian algebras and free associative algebras and obtain some interesting new results.
\end{abstract}

Keywords. Automorphisms of free and polynomial algebras, tame automorphisms, wild automorphisms, coordinates in free algebras

\section{Introduction}

Let $K$ be a field of characteristic 0 and let $X=\left\{x_{1}, \ldots, x_{n}\right\}, n \geq 2$, be a finite set. We denote by $K[X]$ the polynomial algebra in the set of variables $X$ and by $K\langle X\rangle$ the free associative algebra (or the algebra of polynomials in the set $X$ of noncommuting variables) with the same set of free generators. For small $n$ we shall denote the free generators also by $x, y$, etc. We write the automorphisms of $K[X]$ and $K\langle X\rangle$ as $n$-tuples of the images of the coordinates, and $\varphi=\left(f_{1}, \ldots, f_{n}\right)$ means that $\varphi\left(x_{j}\right)=f_{j}(X)=f_{j}\left(x_{1}, \ldots, x_{n}\right)$, $j=1, \ldots, n$. Also, the product $\varphi \psi$ of the automorphisms $\varphi$ and $\psi$ acts on $u(X)$ by $(\varphi \psi)(u)=\varphi(\psi(u))$. An automorphism of $K[X]$ or $K\langle X\rangle$ is called elementary if it is of the form

$$
\left(x_{1}, \ldots, x_{j-1}, \alpha x_{j}+f\left(x_{1}, \ldots, x_{j-1}, x_{j+1}, \ldots, x_{n}\right), x_{j+1}, \ldots, x_{n}\right), \quad \alpha \in K^{*},
$$

and the polynomial $f\left(x_{1}, \ldots, x_{j-1}, x_{j+1}, \ldots, x_{n}\right)$ does not depend on the variable $x_{j}$. The automorphisms belonging to the group generated by elementary automorphisms are

V. Drensky: Institute of Mathematics and Informatics, Bulgarian Academy of Sciences, 1113 Sofia, Bulgaria; e-mail: drensky@math.bas.bg

J.-T. Yu: Department of Mathematics, The University of Hong Kong, Hong Kong SAR, CHINA e-mail: yujt@hkucc.hku.hk

Mathematics Subject Classification (2000): Primary 16S10; Secondary 16W20, 13B10, 13B25 
called tame, all the others are wild. A polynomial $p \in K[X]$ is called a coordinate if it is an automorphic image of $x_{1}$. Moreover, a coordinate $p \in K[X]$ is called tame if there exists a tame automorphism $\varphi \in$ Aut $K[X]$ such that $\varphi\left(x_{1}\right)=p$, otherwise $p$ is called a wild coordinate. One defines in a similar way the coordinates and tame and wild coordinates of $K\langle X\rangle$ and other relatively free algebras. In noncommutative algebra coordinates are often called primitive elements. Obviously, the existence of wild coordinates implies the existence of wild automorphisms, but not vice versa in general.

Problems concerning automorphisms of free objects are similar for free groups, polynomial algebras, free associative and free Lie algebras, and relatively free groups and algebras (see the recent book [MSY] by Mikhalev, Shpilrain, and Yu). One of the most important problems in the theory of automorphisms of polynomial and free associative algebras is whether all automorphisms of $K[X]$ and $K\langle X\rangle$ are tame. The answer is in the affirmative for $K[x, y]$ (Jung-van der Kulk [J, $\mathrm{K}]$ ) and for $K\langle x, y\rangle$ (CzerniakiewiczMakar-Limanov [Cz, ML1, ML2]). In 1970, Nagata [N] constructed his famous automorphism of $K[x, y, z]$ which is wild as an automorphism fixing $z$ and conjectured that it is also wild as a usual automorphism of $K[x, y, z]$. More than 30 years later Shestakov and Umirbaev [SU1, SU2, SU3], using methods of Poisson brackets, degree estimations and peak reduction, proved the Nagata Conjecture, in particular, they proved that the Nagata automorphism is wild. Umirbaev and J.-T. Yu [UY] proved the Strong Nagata Conjecture, namely, there exist wild coordinates of $K[x, y, z]$. In particular, the two nontrivial Nagata coordinates are both wild. There were also attempts, unfortunately unsuccessful, to lift the Nagata automorphism to an automorphism of $K\langle x, y, z\rangle$ (see, for instance, our paper with Gutierrez [DGY]). (A lifting of the Nagata automorphism would provide immediately a wild automorphism of $K\langle x, y, z\rangle$.) There is another automorphism of $K\langle x, y, z\rangle$, suggested by Anick,

$$
(x+y(x y-y z), y, z+(x y-y z) y) \in \text { Aut } K\langle x, y, z\rangle
$$

(see the book by Cohn [C2, p. 343]), which was suspected to be wild, although it induces a tame automorphism of $K[x, y, z]$. Exchanging the places of $y$ and $z$ in the above automorphism, we replace it with the automorphism

$$
\omega=(x+z(x z-z y), y+(x z-z y) z, z) .
$$

It fixes $z$, and $\omega(x)$ and $\omega(y)$ are linear in $x$ and $y$. From now on, we shall refer to $\omega$ as the Anick automorphism.

Conjecture 1 (Anick Conjecture). There exist wild automorphisms in Aut $K\langle x, y, z\rangle$. In particular, the Anick automorphism is wild.

We established [DY3] that the Anick automorphism is wild in the class of automorphisms fixing the variable $z$, and very recently Umirbaev [U2] has proved the Anick Conjecture, that is, the wildness of the Anick automorphism as an automorphism of $K\langle x, y, z\rangle$.

The work of Nagata $[N]$ motivated the study of automorphisms fixing a variable. More generally, we introduce another set $Z=\left\{z_{1}, \ldots, z_{m}\right\}$ and consider the algebras $K[X, Z]$ and $K\langle X, Z\rangle$, freely generated by the set $X \cup Z$. Studying automorphisms fixing the set $Z$, 
we use the same notation $\varphi=\left(f_{1}, \ldots, f_{n}, z_{1}, \ldots, z_{m}\right)$, meaning that $\varphi\left(x_{j}\right)=f_{j}(X, Z)$, $j=1, \ldots, n$, and $\varphi\left(z_{i}\right)=z_{i}, i=1, \ldots, m$, and denote the corresponding automorphism group by $\operatorname{Aut}_{Z} K[X, Z]$ and $\mathrm{Aut}_{Z} K\langle X, Z\rangle$, respectively. Again, an automorphism is $Z$ elementary if it is of the form $\varphi=\left(x_{1}, \ldots, \alpha x_{j}+f(X, Z), \ldots, x_{n}, z_{1}, \ldots, z_{m}\right)$, and $f(X, Z)$ does not depend on $x_{j}$. The automorphism is tame in the class of automorphisms fixing $Z$ (or $Z$-tame) if it belongs to the group generated by $Z$-elementary automorphisms. In the general case, this group may be smaller than the group generated by $Z$-triangular and $Z$-affine automorphisms because some $Z$-affine automorphisms may not be products of $Z$-elementary ones.

An important consequence of [SU1, SU2, SU3, DY1, DY2] is that every $z$-wild automorphism of $K[x, y, z]$ is a wild automorphism of $K[x, y, z]$. A method of constructing a large class of such automorphisms was given by us in [DY2].

The next step in studying automorphisms of free algebras is to study coordinates, or automorphic images of $x_{1}$. In noncommutative algebra coordinates are also called primitive elements. There are algorithms to recognize coordinates of $K[x, y]$ (Shpilrain and $\mathrm{Yu}$ [SY]) and $z$-coordinates of $K[x, y, z]$ (see our paper [DY2] as well as our survey [DY1]). As a consequence of their proof of the Strong Nagata Conjecture, Umirbaev and J.-T. Yu [UY] established that if $f(x, y, z)$ is a $z$-wild coordinate in $K[x, y, z]$, then it is immediately a wild coordinate in $K[x, y, z]$.

Obviously, results for (commutative) polynomial algebras inspire problems on free associative algebras as there is a natural surjective homomorphism from $K\langle X\rangle$ to $K[X]$ which induces a natural (not necessarily surjective) homomorphism from Aut $K\langle X\rangle$ to Aut $K[X]$. In this paper we shall be interested in the following problem motivated by [UY]:

Problem 1. If $f(X, Z) \in K\langle X, Z\rangle$ is the image of $x_{1}$ under a $Z$-wild automorphism, is there a tame automorphism (maybe not fixing $Z$ ) which also sends $x_{1}$ to $f(X, Z)$ ?

If such a tame automorphism does not exist, then we call $f(X, Z)$ a wild coordinate of $K\langle X, Z\rangle$.

As an analog of the Strong Nagata Conjecture in [UY], we state

Conjecture 2 (Strong Anick Conjecture). There exist wild coordinates in $K\langle x, y, z\rangle$. In particular, the two nontrivial coordinates of the Anick automorphism are both wild.

The Anick automorphism has the property that it fixes $z$, and $\omega(x)$ and $\omega(y)$ are linear in $x$ and $y$. In our paper [DY3] we showed that such an automorphism of $K\langle x, y, z\rangle$ is $z$-tame if and only if a certain $2 \times 2$ matrix with entries from $K\left[z_{1}, z_{2}\right]$ is a product of elementary matrices. This idea has been used by Umirbaev [U2] in the final step of his proof of the wildness of the Anick automorphism. Now we show that if $f(x, y, z)$ is a wild $z$-coordinate in $K\langle x, y, z\rangle$, and $f(x, y, z)$ is linear in $x, y$, then $f(x, y, z)$ is also wild in the sense of Problem 11 This is one of the main results of the paper. It immediately confirms the Strong Anick Conjecture.

The class of wild automorphisms of $K\langle x, y, z\rangle$ discovered by Umirbaev [U2] is larger than the class of $z$-wild automorphisms $(f, g, z)$ such that the polynomials $f, g$ are linear 
in $x, y$. Our method also shows that all automorphisms of Umirbaev's class have the property that at least two of their coordinates are wild. The same result holds for another large class of automorphisms of $K\langle x, y, z\rangle$ which is not covered by Umirbaev [U2].

Our main result suggests an algorithm deciding whether a polynomial $f(x, y, z) \in$ $K\langle x, y, z\rangle$ which is linear in $x$ and $y$, is a tame coordinate. If it is, then the algorithm shows how to find a product of $z$-elementary automorphisms which sends $x$ to $f(x, y, z)$. (Of course, in all algorithmic considerations we assume that the ground field $K$ is constructive, and we may perform calculations there.) In this part of the paper we use the approach and the results of Umirbaev [U2], combined with our approach from [DY3].

On the other hand, we show that the situation is completely different in the case of the free metabelian algebra $M(x, y, z)$. We construct an automorphism which fixes $y$ and $z$ and cannot be lifted to an automorphism of $K\langle x, y, z\rangle$. The proof is based on a test recognizing some classes of endomorphisms which are not automorphisms. This test originates from group theory (see Bryant, Gupta, Levin and Mochizuki [BGLM]) and was adapted to algebras by Bryant and Drensky $[\overline{\mathrm{BD}}]$.

In addition, we show that an automorphism of $K[X, Z]$ or $K\langle X, Z\rangle$ which is $Z$-wild cannot be lifted to a $Z$-automorphism of the absolutely free algebra $K\{X, Z\}$. (As a consequence of a result of Kurosh $[\overline{\mathrm{Ku}}]$, all automorphisms of the absolutely free algebra are tame.) This is equivalent to the fact that there exist no $Z$-wild automorphisms of $K\{X, Z\}$.

\section{Proof of main results}

Dicks and Lewin [DL] introduced the Jacobian matrix of an endomorphism of $K\langle X\rangle$. This is an $n \times n$ matrix with entries from the tensor product $K\langle X\rangle \otimes_{K} K\langle X\rangle^{\text {op }}$ of the free algebra $K\langle X\rangle$ and its opposite (or anti-isomorphic) algebra $K\langle X\rangle^{\text {op }}$. For $n=2$ they proved that the Jacobian matrix is invertible over $K\langle x, y\rangle \otimes_{K} K\langle x, y\rangle^{\text {op }}$ if and only if the endomorphism is an automorphism. The general case of any $n$ was established by Schofield $[\mathbf{S c}]$; this is the Jacobian Conjecture for free associative algebras. The partial derivatives and the Jacobian matrix of Dicks and Lewin can be defined as follows:

$$
\frac{\partial x_{i}}{\partial x_{i}}=1, \quad \frac{\partial x_{j}}{\partial x_{i}}=0, \quad j \neq i
$$

and, for a monomial $w=x_{i_{1}} \cdots x_{i_{m}} \in K\langle X\rangle$,

$$
\frac{\partial w}{\partial x_{i}}=\sum_{k=1}^{m}\left(x_{i_{1}} \cdots x_{i_{k-1}}\right) \otimes\left(x_{i_{k+1}} \cdots x_{i_{m}}\right) \frac{\partial x_{i_{k}}}{\partial x_{i}},
$$

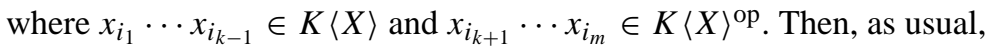

$$
J(\varphi)=\left(\frac{\partial \varphi\left(x_{j}\right)}{\partial x_{i}}\right), \quad \varphi \in \operatorname{End} K\langle X\rangle .
$$

We need the following lemma. 
Lemma 2.1. The only automorphisms of $K\langle X\rangle$ fixing $x_{2}, \ldots, x_{n}$ are the tame automorphisms of the form

$$
\tau=\left(\alpha x_{1}+f\left(x_{2}, \ldots, x_{n}\right), x_{2}, \ldots, x_{n}\right), \quad \alpha \in K^{*}, f\left(x_{2}, \ldots, x_{n}\right) \in K\left\langle x_{2}, \ldots, x_{n}\right\rangle .
$$

Proof. The shortest way to establish the lemma is to use the invertibility of the Jacobian matrix. Let $\tau=\left(g(X), x_{2}, \ldots, x_{n}\right) \in$ Aut $K\langle X\rangle$ fix $x_{2}, \ldots, x_{n}$. Then the matrix

$$
J(\tau)=\left(\begin{array}{cccc}
\frac{\partial g}{\partial x_{1}} & 0 & \ldots & 0 \\
\frac{\partial g}{\partial x_{2}} & 1 & \ldots & 0 \\
\vdots & \vdots & \ddots & \vdots \\
\frac{\partial g}{\partial x_{n}} & 0 & \ldots & 1
\end{array}\right)
$$

is invertible over $K\langle X\rangle \otimes_{K} K\langle X\rangle^{\text {op }}$ and this implies that $\partial g / \partial x_{1}$ is equal to a nonzero constant $\alpha$. Hence the only term of $g(X)$ depending on $x_{1}$ is $\alpha x_{1}$.

For $K\langle x, y, z\rangle$, the endomorphisms which fix $z$ and are linear in $x$ and $y$ are of the form $\rho=(f(x, y, z), g(x, y, z), z)$, where

$$
\begin{aligned}
& f(x, y, z)=\sum_{p, q \geq 0} \alpha_{p q} z^{p} x z^{q}+\sum_{p, q \geq 0} \beta_{p q} z^{p} y z^{q}+f_{0}(z), \\
& g(x, y, z)=\sum_{p, q \geq 0} \gamma_{p q} z^{p} x z^{q}+\sum_{p, q \geq 0} \delta_{p q} z^{p} y z^{q}+g_{0}(z),
\end{aligned}
$$

$\alpha_{p q}, \beta_{p q}, \gamma_{p q}, \delta_{p q} \in K$, and $f_{0}(z), g_{0}(z)$ are polynomials in $z$. Applying the Jacobian matrix of Dicks and Lewin in this concrete case, in [DY3] we obtained:

Proposition 2.2. (i) The endomorphism $\rho=(f(x, y, z), g(x, y, z), z)$ which fixes $z$ and is linear in $x$ and $y$ is an automorphism if and only if the $2 \times 2$ matrix

$$
J_{z}(\rho)=\left(\begin{array}{ll}
\sum_{p, q \geq 0} \alpha_{p q} z_{1}^{p} z_{2}^{q} & \sum_{p, q \geq 0} \gamma_{p q} z_{1}^{p} z_{2}^{q} \\
\sum_{p, q \geq 0} \beta_{p q} z_{1}^{p} z_{2}^{q} & \sum_{p, q \geq 0} \delta_{p q} z_{1}^{p} z_{2}^{q}
\end{array}\right)
$$

with entries from $K\left[z_{1}, z_{2}\right]$ is invertible. All such automorphisms induce z-tame automorphisms of $K[x, y, z]$.

(ii) The automorphism $\rho$ is $z$-tame if and only if the matrix $J_{z}(\rho)$ belongs to the group generated by elementary matrices with entries from $K\left[z_{1}, z_{2}\right]$.

For example, for the Anick automorphism,

$$
J_{z}(\omega)=\left(\begin{array}{cc}
1+z_{1} z_{2} & z_{2}^{2} \\
-z_{1}^{2} & 1-z_{1} z_{2}
\end{array}\right)
$$

and by a result of Cohn [C1], the matrix $J_{z}(\omega)$ cannot be represented as a product of elementary matrices. 
Let $Z=\left\{z_{1}, \ldots, z_{m}\right\}$. We denote by $G E_{2}(K[Z])$ the subgroup of $G L_{2}(K[Z])$ generated by the diagonal and by the elementary matrices

$$
\left(\begin{array}{cc}
\alpha_{1} & 0 \\
0 & \alpha_{2}
\end{array}\right), \quad\left(\begin{array}{cc}
1 & f(Z) \\
0 & 1
\end{array}\right), \quad\left(\begin{array}{cc}
1 & 0 \\
f(Z) & 1
\end{array}\right)
$$

with entries from $K[Z]$. There is an algorithm deciding whether a matrix in $G L_{2}(K[Z])$ belongs to $G E_{2}(K[Z])$. It was suggested by Tolhuizen, Hollmann, and Kalker [THK] for the partial ordering by degree and, independently, by Park [P1, [P2] for any monomial ordering on $K[Z]$. One applies Gaussian elimination process to the matrix based on the Euclidean division algorithm for $K[Z]$. The matrix belongs to $G E_{2}(K[Z])$ if and only if this procedure brings it to the identity matrix. For our purposes, we need the following version of the Euclidean algorithm. If $a(Z), b(Z)$ are two nonzero polynomials with homogeneous components of maximal degree $\overline{a(Z)}, \overline{b(Z)}$, respectively, then the Euclidean algorithm can be applied to $a(Z)$ and $b(Z)$ if $\overline{a(Z)}=\overline{b(Z)} q(Z)$ for some $q(Z) \in K[Z]$ (or $\overline{b(Z)}=\overline{a(Z)} q(Z)$ ) when we replace $a(Z)$ with $a(Z)-b(Z) q(Z)$ (or, respectively, we replace $b(Z)$ with $b(Z)-a(Z) q(Z)$ ). In matrix form, these operations correspond, respectively, to

$$
\begin{aligned}
\left(\begin{array}{c}
a(Z)-b(Z) q(Z) \\
b(Z)
\end{array}\right) & =\left(\begin{array}{cc}
1 & -q(Z) \\
0 & 1
\end{array}\right)\left(\begin{array}{l}
a(Z) \\
b(Z)
\end{array}\right), \\
\left(\begin{array}{c}
a(Z) \\
b(Z)-a(Z) q(Z)
\end{array}\right) & =\left(\begin{array}{cc}
1 & 0 \\
-q(Z) & 1
\end{array}\right)\left(\begin{array}{l}
a(Z) \\
b(Z)
\end{array}\right) .
\end{aligned}
$$

For us, the most convenient form of the result in [P1, $[\mathrm{P} 2, \mathrm{THK}]$ is as stated in [THK].

Proposition 2.3. Let $a(Z), b(Z)$ be two polynomials in $K[Z]$. Then there exist $c(Z), d(Z) \in K[Z]$ such that the matrix

$$
G=\left(\begin{array}{ll}
a(Z) & c(Z) \\
b(Z) & d(Z)
\end{array}\right)
$$

belongs to $G E_{2}(K[Z])$ if and only if we can bring the pair $(a(Z), b(Z))$ to $(\alpha, 0), 0 \neq$ $\alpha \in K$, using the Euclidean algorithm only.

Clearly, in this case the equations (1) and (2) give the decomposition of $G$ as a product of elementary matrices.

We need a description of the free metabelian associative algebra and a short exposition of the results of Umirbaev. Recall that the free metabelian algebra

$$
M(X)=K\langle X\rangle /\left(\left[t_{1}, t_{2}\right]\left[t_{3}, t_{4}\right]\right)^{T}
$$

is the relatively free algebra of rank $n$ in the variety of associative algebras defined by the polynomial identity $\left[t_{1}, t_{2}\right]\left[t_{3}, t_{4}\right]=0$. In order to define partial derivatives and the Jacobian matrix of an endomorphism, we need two more sets of variables $U=\left\{u_{1}, \ldots, u_{n}\right\}$ 
and $V=\left\{v_{1}, \ldots, v_{n}\right\}$ of the same cardinality as $X$. We consider the polynomial algebra $K[U, V]$. Changing a little the notation of Umirbaev [U1], we define formal partial derivatives $\partial_{M} / \partial_{M} x_{i}$ assuming that

$$
\frac{\partial_{M} x_{i}}{\partial_{M} x_{i}}=1, \quad \frac{\partial_{M} x_{j}}{\partial_{M} x_{i}}=0, \quad j \neq i,
$$

and, for a monomial $w=x_{i_{1}} \cdots x_{i_{m}} \in M(X)$,

$$
\frac{\partial_{M} w}{\partial_{M} x_{i}}=\sum_{k=1}^{m} u_{i_{1}} \cdots u_{i_{k-1}} v_{i_{k+1}} \ldots v_{i_{m}} \frac{\partial_{M} x_{i_{k}}}{\partial_{M} x_{i}} .
$$

These are the homomorphic images of the partial derivatives of Dicks and Lewin under the natural homomorphism $K\langle X\rangle \otimes_{K} K\langle X\rangle^{\text {op }} \rightarrow K[U, V]$ which sends $x_{i} \otimes 1$ and $1 \otimes x_{j}$ to $u_{i}$ and $v_{j}$, respectively. A polynomial $f(X) \in M(X)$ belongs to the commutator ideal of $M(X)$, i.e., to the kernel of the natural homomorphism $M(X) \rightarrow K[X]$, if and only if

$$
\sum_{i=1}^{n}\left(u_{i}-v_{i}\right) \frac{\partial_{M} f}{\partial_{M} x_{i}}=0 .
$$

The Jacobian matrix of an endomorphism $\varphi$ of $M(X)$ is

$$
J_{M}(\varphi)=\left(\frac{\partial_{M} \varphi\left(x_{j}\right)}{\partial_{M} x_{i}}\right),
$$

which is a matrix with entries from $K[U, V]$. One of the main results in [U1] is that the Jacobian matrix $J_{M}(\varphi)$ is invertible (as a matrix with entries from $K[U, V]$ ) if and only if $\varphi$ is an automorphism of $M(X)$. Clearly, the invertibility of $J_{M}(\varphi)$ is equivalent to $0 \neq \operatorname{det}\left(J_{M}(\varphi)\right) \in K$. In this section we shall work with free algebras of rank 3 only and shall assume that the sets $X, U, V$ are, respectively,

$$
X=\{x, y, z\}, \quad U=\left\{x_{1}, y_{1}, z_{1}\right\}, \quad V=\left\{x_{2}, y_{2}, z_{2}\right\} .
$$

Let $T(K\langle x, y, z\rangle), T(M(x, y, z))$ and $T(K[x, y, z])$ be, respectively, the groups of tame automorphisms of $K\langle x, y, z\rangle, M(x, y, z)$, and $K[x, y, z]$. There is a natural homomorphism

$$
T(K\langle x, y, z\rangle) \rightarrow T(M(x, y, z)) \rightarrow T(K[x, y, z]) .
$$

Let $\operatorname{Ker}(\pi)$ be the kernel of $\pi: T(M(x, y, z)) \rightarrow T(K[x, y, z])$. Further developing the methodology in [SU1, SU2, SU3], Umirbaev [U2] discovered the defining relations of $T(K[x, y, z])$. As a consequence, he proved the following.

Proposition 2.4. As a normal subgroup of $T(M(x, y, z))$, the kernel of $\pi$ is generated by the automorphisms

$$
\psi=(x+f(y, z), y, z), \quad f(y, z)=\sum_{p, q, r, s \geq 0} \alpha_{p q r s} y^{p} z^{q}[y, z] y^{r} z^{s}, \quad \alpha_{p q r s} \in K .
$$


Moreover, any tame automorphism $\vartheta$ from $\operatorname{Ker}(\pi)$ has a Jacobian matrix which is a product of elementary matrices. The next key observation of Umirbaev is the following. Let $\vartheta$ be any automorphism from the kernel of the natural homomorphism Aut $M(x, y, z) \rightarrow$ Aut $K[x, y, z]$. Then

$$
J_{M}(\vartheta)=J_{M}(\vartheta)\left(x_{1}, y_{1}, z_{1}, x_{2}, y_{2}, z_{2}\right)
$$

is a $3 \times 3$ matrix with entries from $K\left[x_{1}, y_{1}, z_{1}, x_{2}, y_{2}, z_{2}\right]$. If we replace $x_{1}, y_{1}, x_{2}, y_{2}$ with zeros, then the matrix $J_{M}(\vartheta)\left(0,0, z_{1}, 0,0, z_{2}\right)$ will be of the form

$$
\overline{J_{M}(\vartheta)}\left(z_{1}, z_{2}\right)=\left(\begin{array}{ccc}
1+w_{11} & w_{12} & w_{13} \\
w_{21} & 1+w_{22} & w_{23} \\
0 & 0 & 1
\end{array}\right),
$$

where the polynomials $w_{i j}=w_{i j}\left(z_{1}, z_{2}\right)$ have no constant terms. Define the $2 \times 2$ matrix

$$
J_{2}(\vartheta)\left(z_{1}, z_{2}\right)=\left(\begin{array}{cc}
1+w_{11}\left(z_{1}, z_{2}\right) & w_{12}\left(z_{1}, z_{2}\right) \\
w_{21}\left(z_{1}, z_{2}\right) & 1+w_{22}\left(z_{1}, z_{2}\right)
\end{array}\right) .
$$

Proposition 2.5 (Umirbaev [U2]). If $\vartheta \in \operatorname{Ker}(\pi)$, then $J_{2}(\vartheta)\left(z_{1}, z_{2}\right)$ is a product of elementary matrices with entries from $K\left[z_{1}, z_{2}\right]$.

Note that the matrix $J_{2}(\bar{\rho})$ of the automorphism $\bar{\rho}$ of $M(x, y, z)$ induced by the automorphism $\rho$ of $K\langle x, y, z\rangle$ coincides with the matrix $J_{z}(\rho)$, the Jacobian matrix of $(\rho(x), \rho(y))$, when $\rho$ fixes $z$ and is linear with respect to $x, y$.

Now we are ready to prove the main results in this article.

Theorem 2.6. Let $K$ be a field of characteristic 0 and let the polynomial $f(x, y, z) \in$ $K\langle x, y, z\rangle$ be linear in $x, y$. If there exists a wild automorphism of $K\langle x, y, z\rangle$ which fixes $z$ and sends $x$ to $f(x, y, z)$, then every automorphism of $K\langle x, y, z\rangle$ which sends $x$ to $f(x, y, z)$ is also wild. So, $f(x, y, z)$ is a wild coordinate of $K\langle x, y, z\rangle$.

Proof. Let $\sigma=(f(x, y, z), h(x, y, z), z)$ be a wild automorphism of $K\langle x, y, z\rangle$ which fixes $z$ and sends $x$ to $f(x, y, z)$. We write $f(x, y, z)$ in the form

$$
f(x, y, z)=\sum_{p, q \geq 0} \alpha_{p q} z^{p} x z^{q}+\sum_{p, q \geq 0} \beta_{p q} z^{p} y z^{q}+f_{0}(z),
$$

where $\alpha_{p q}, \beta_{p q} \in K$, and $f_{0}(z)$ is a polynomial in $z$. Let

$$
a\left(z_{1}, z_{2}\right)=\sum_{p, q \geq 0} \alpha_{p q} z_{1}^{p} z_{2}^{q}, \quad b\left(z_{1}, z_{2}\right)=\sum_{p, q \geq 0} \beta_{p q} z_{1}^{p} z_{2}^{q} .
$$

First we shall show that the polynomials $a\left(z_{1}, z_{2}\right), b\left(z_{1}, z_{2}\right)$ cannot constitute the first column of a matrix from $G E_{2}\left(K\left[z_{1}, z_{2}\right]\right)$. Suppose, on the contrary,

$$
\begin{gathered}
J=\left(\begin{array}{ll}
a\left(z_{1}, z_{2}\right) & c\left(z_{1}, z_{2}\right) \\
b\left(z_{1}, z_{2}\right) & d\left(z_{1}, z_{2}\right)
\end{array}\right) \in G E_{2}\left(K\left[z_{1}, z_{2}\right]\right), \\
c\left(z_{1}, z_{2}\right)=\sum_{p, q \geq 0} \gamma_{p q} z_{1}^{p} z_{2}^{q}, \quad d\left(z_{1}, z_{2}\right)=\sum_{p, q \geq 0} \delta_{p q} z_{1}^{p} z_{2}^{q} .
\end{gathered}
$$


Consider the polynomial

$$
g(x, y, z)=\sum_{p, q \geq 0} \gamma_{p q} z^{p} x z^{q}+\sum_{p, q \geq 0} \delta_{p q} z^{p} y z^{q} .
$$

By Proposition 2.2 ii), the automorphism $\rho=(f(x, y, z), g(x, y, z), z)$ is tame in the group of automorphisms fixing $z$. Hence the automorphism $\rho^{-1} \sigma$ is also wild. But

$$
\rho^{-1} \sigma=(x, k(x, y, z), z)
$$

for some $k(x, y, z) \in K\langle x, y, z\rangle$. This contradicts Lemma 2.1

Hence $a\left(z_{1}, z_{2}\right), b\left(z_{1}, z_{2}\right)$ cannot constitute the first column of a matrix from $G E_{2}\left(K\left[z_{1}, z_{2}\right]\right)$.

The next step is to produce a wild automorphism of $K\langle x, y, z\rangle$ which induces the identity automorphism of $K[x, y, z]$.

Let $\sigma=(f(x, y, z), h(x, y, z), z)$ be the above wild automorphism of $K\langle x, y, z\rangle$ which fixes $z$ and sends $x$ to $f(x, y, z)$, and let $h_{1}(x, y, z)$ be the component of $h$ which is linear with respect to $x, y$. Then $\tau=\left(f(x, y, z), h_{1}(x, y, z), z\right)$ is also a wild automorphism of $K\langle x, y, z\rangle$ which induces a $z$-tame automorphism of $K[x, y, z]$. (The automorphism $\tau$ is wild since $\sigma^{-1} \tau$ sends $x$ to $x$ and $z$ to $z$, so by Lemma 2.1, $\sigma^{-1} \tau$ is tame. The induced automorphism is $z$-tame by Proposition 2.2.) Let $\psi$ be the corresponding $z$-tame automorphism of $K\langle x, y, z\rangle$, linear in $x, y$. Then $\widetilde{\tau}=\psi^{-1} \tau$ is still wild and induces the identity automorphism of $K[x, y, z]$.

Now, let $\varphi$ be any tame automorphism of $K\langle x, y, z\rangle$ which sends $x$ to $f(x, y, z)$. Replacing $\varphi$ with $\widetilde{\varphi}=\psi^{-1} \varphi$, we obtain a tame automorphism for which $\widetilde{\varphi}(x)=\tilde{\tau}(x)$.

The automorphism $\tilde{\varphi}$ induces a tame automorphism of $K[x, y, z]$ which fixes $x$. By results in [DY1, DY2, SU1, SU3], such an automorphism is tame in the class of automorphisms fixing $x$ and we can lift it to an $x$-tame automorphism $\theta$ of $K\langle x, y, z\rangle$. So we obtain a tame automorphism $\widehat{\varphi}=\widetilde{\varphi} \theta^{-1}$ which induces the identity automorphism of $K[x, y, z]$ and $\widehat{\varphi}(x)=\tilde{\tau}(x)$.

Let $\xi$ be the automorphism of $M(x, y, z)$ induced by $\widehat{\varphi}$. It is in the kernel of the homomorphism $\pi$ of Aut $M(x, y, z) \rightarrow$ Aut $K[x, y, z]$. The first columns of the matrices $J_{2}(\xi)$ and $J_{2}(\pi(\tilde{\tau}))$ coincide. As remarked above, this column cannot be a column of a matrix from $G E_{2}\left(K\left[z_{1}, z_{2}\right]\right)$ since $\tilde{\tau}$ is wild. On the other hand, by Proposition 2.5 it is a column of a matrix from $G E_{2}\left(K\left[z_{1}, z_{2}\right]\right)$. This contradiction completes the proof.

Theorem 2.6 and Proposition 2.3 give an algorithm deciding whether a polynomial $f(x, y, z) \in K\langle x, y, z\rangle$ which is linear in $x$ and $y$, is a tame coordinate. If it is, then the algorithm finds a product of $z$-elementary automorphisms which sends $x$ to $f(x, y, z)$.

The following consequence of Theorem 2.6 proves the Strong Anick Conjecture.

Theorem 2.7. The Strong Anick Conjecture is true. Namely, there exist wild coordinates in $K\langle x, y, z\rangle$. In particular, the two nontrivial coordinates $x+z(x z-z y)$ and $y+(x z-$ $z y) z$ of the Anick automorphism

$$
\omega=(x+z(x z-z y), y+(x z-z y) z, z)
$$

are both wild. 
Proof. The partial derivatives of $f(x, y, z)=\omega(x)=x+z(x z-z y)$ are

$$
a\left(z_{1}, z_{2}\right)=\frac{\partial f}{\partial x}=1+z_{1} z_{2}, \quad b\left(z_{1}, z_{2}\right)=\frac{\partial f}{\partial y}=-z_{1}^{2} .
$$

Since we cannot apply the Euclidean algorithm to $a\left(z_{1}, z_{2}\right)$ and $b\left(z_{1}, z_{2}\right)$, Theorem 2.6 implies that $f(x, y, z)$ is a wild coordinate.

We call an automorphism $\varphi=(f(x, y, z), g(x, y, z), z)$ of $K\langle x, y, z\rangle$ Anick-like if $f(x, y, z)$ and $g(x, y, z)$ are linear in $x, y$ and the matrix $J_{z}(\varphi)$ does not belong to $G E_{2}\left(K\left[z_{1}, z_{2}\right]\right)$. The following corollary is an analogue of a result from [UY].

Corollary 2.8. The two nontrivial coordinates $f(x, y, z), g(x, y, z)$ of any Anick-like automorphism

$$
\varphi=(f(x, y, z), g(x, y, z), z)
$$

of $K\langle x, y, z\rangle$ are wild.

Proof. Let

$$
\frac{\partial f}{\partial x}=a\left(z_{1}, z_{2}\right), \quad \frac{\partial f}{\partial y}=b\left(z_{1}, z_{2}\right) .
$$

We cannot apply the Euclidean algorithm to bring the pair $\left(a\left(z_{1}, z_{2}\right), b\left(z_{1}, z_{2}\right)\right)$ to $(\alpha, 0)$, $0 \neq \alpha \in K$, because $J_{z}(\varphi) \notin G E_{2}\left(K\left[z_{1}, z_{2}\right]\right)$. Hence Theorem 2.6 shows that $f(x, y, z)$ is a wild coordinate. Similar arguments work for $g(x, y, z)$.

In the spirit of the above results, we obtain the following theorem which is much stronger.

Theorem 2.9. Let $f(x, y, z)$ be a z-coordinate of $K\langle x, y, z\rangle$ without terms depending only on $z$ (i.e. $f(0,0, z)=0$ ). If the linear part (with respect to $x$ and $y) f_{1}(x, y, z)$ of $f(x, y, z)$ is a $z$-wild coordinate, then $f(x, y, z)$ itself is also a wild coordinate of $K\langle x, y, z\rangle$.

Proof. Since $f(x, y, z)$ is a $z$-coordinate of $K\langle x, y, z\rangle$, there exists a $z$-automorphism $\sigma=(f(x, y, z), g(x, y, z), z)$ of $K\langle x, y, z\rangle$. Obviously we may assume $g(0,0, z)=0$ (otherwise just replace $g(x, y, z)$ by $(g(x, y, z)-g(0,0, z))$. Let $\sigma_{1}=\left(f_{1}(x, y, z)\right.$, $\left.g_{1}(x, y, z), z\right)$ be the automorphism which is the linear part of $\sigma$. By assumption $\sigma_{1}$ is a wild automorphism. We have to prove the wildness of all automorphisms $\varphi=$ $(f(x, y, z), u(x, y, z), v(x, y, z))$ of $K\langle x, y, z\rangle$ with first coordinate equal to $f(x, y, z)$. Consider the automorphisms $\bar{\sigma}=(\bar{f}, \bar{g}, z)$ and $\bar{\varphi}=(\bar{f}, \bar{u}, \bar{v})$ of $K[x, y, z]$ induced by $\sigma$ and $\varphi$, respectively. If $\bar{\sigma}$ is wild, then, by the theorem of Umirbaev and Yu [UY], $\bar{f}$ is a wild coordinate of $K[x, y, z]$. Hence $\bar{\varphi}$ is a wild automorphism of $K[x, y, z]$. This implies that $\varphi$ is a wild automorphism of $K\langle x, y, z\rangle$ and therefore $f(x, y, z)$ is a wild coordinate. Hence we may assume that $\bar{\sigma}$ is a tame automorphism of $K[x, y, z]$.

Now we suppose that the automorphism $\varphi$ is tame and repeat the main steps of the proof of Theorem 2.6. Since $\bar{\sigma}$ is tame, by [DY1, DY3, SU1, [SU3] it is also $z$-tame. Let $\psi$ be some $z$-tame automorphism of $K\langle x, y, z\rangle$ which induces $\bar{\sigma}$ and let $\psi_{1}$ be the linear part of $\psi$. Replacing $\sigma$ with $\widetilde{\sigma}=\psi^{-1} \sigma$ and $\varphi$ with $\widetilde{\varphi}=\psi^{-1} \varphi$, we find that 
the tame automorphism $\widetilde{\varphi}$ fixes $x$ modulo the commutator ideal of $K\langle x, y, z\rangle$. Since $\widetilde{\sigma}$ is a composition of the $z$-automorphisms $\psi^{-1}$ and $\sigma$, its linear part $(\widetilde{\sigma})_{1}$ is also a $z$ automorphism which is equal to the composition $\psi_{1}^{-1} \varphi_{1}$ of the linear components of $\psi_{1}^{-1}$ and $\varphi_{1}$. Hence $(\widetilde{\sigma})_{1}$ is wild and we may reduce our considerations to the case when $\tilde{\sigma}(x)=f(x, y, z)$ is congruent to $x$ modulo the commutator ideal of $K\langle x, y, z\rangle$. Since $\tilde{\varphi}$ induces a tame automorphism of $K[x, y, z]$, by [DY1, DY3, [SU1, SU3] again, the induced automorphism is also $x$-tame and we can lift it to an $x$-tame automorphism $\theta$ of $K\langle x, y, z\rangle$. The tame automorphism $\widehat{\varphi}=\widetilde{\varphi} \theta^{-1}$ induces the identity automorphism of $K[x, y, z]$ and $\widehat{\varphi}(x)=f(x, y, z)$. Now, as in Theorem 2.6, the proof is completed by considerations in the free metabelian algebra $M(x, y, z)$.

Remark 2.10. The restriction $f(0,0, z)=0$ is essential for the proof of Theorem 2.9 (note that obviously we may assume $g(0,0, z)=0$, otherwise just replace $g(x, y, z)$ by $g(x, y, z)-g(0,0, z))$. We use it when, modifying simultaneously the automorphisms $\sigma=(f(x, y, z), g(x, y, z), z)$ and $\varphi=(f(x, y, z), u(x, y, z), v(x, y, z))$ of $K\langle x, y, z\rangle$, we bring $\sigma$ and $\varphi$ to automorphisms which send $x$ to the same element congruent to $x$ modulo the commutator ideal, still keeping the property that the linear component of the image of $x$ is wild. Nevertheless, it seems very unlikely to have a wild automorphism $(f, g, z)$ with $f(0,0, z)=0$ such that $f(x, y, z)+a(z)$ is a tame coordinate for some polynomial $a(z)$ in view of the next theorem.

Theorem 2.11. Let $(f, g, z)$ be an automorphism of $K\langle x, y, z\rangle$ and let the linear part (with respect to $x$ and $y)$ of it, $\left(f_{1}, g_{1}, z\right)$, be a z-wild automorphism. Then $(f, g, z)$ is also a wild automorphism of $K\langle x, y, z\rangle$.

Proof. Let $f(x, y, z)=f^{\prime}(x, y, z)+f_{0}(z), g(x, y, z)=g^{\prime}(x, y, z)+g_{0}(z)$, where $f^{\prime}, g^{\prime}$ do not contain monomials depending on $z$ only. Define the automorphism $\tau=$ $\left(x-f_{0}(z), y-g_{0}(z), z\right)$. Then the automorphism $\sigma=(f, g, z)$ is tame (or $z$-tame) if and only if $\sigma \tau=\left(f^{\prime}, g^{\prime}, z\right)$ is tame (or $z$-tame). Since the polynomials $f, f^{\prime}$ and $g, g^{\prime}$ have the same linear components $f_{1}$ and $g_{1}$, we apply Theorem 2.9 .

Remark 2.12. The above theorem is much stronger than the main result in [U2] where only the automorphisms linear with respect to $x$ and $y$ are dealt with.

The following example gives a large class of wild automorphisms and wild coordinates. It is based on the polynomial $x z-z y$ which appears in the Anick automorphism.

Example 2.13. Let $h(t, z) \in K\langle t, z\rangle$ and let $h(0,0)=0$. Then

$$
\sigma_{h}=(x+z h(x z-z y, z), y+h(x z-z y, z) z, z)
$$

is an automorphism of $K\langle x, y, z\rangle$ fixing $x z-z y$. If the linear component (with respect to $x, y) h_{1}(x z-z y, z)$ of $h(x z-z y, z)$ is not equal to 0 , then this automorphism belongs to the class of wild automorphisms in Theorem 2.9 . As $\left(\sigma_{h}\right)_{1}=\left(x+z h_{1}(x z-z y, z), y+\right.$ $\left.h_{1}(x z-z y, z) z, z\right)$ is an automorphism of $K\langle x, y, z\rangle$ and its matrix $J_{z}\left(\left(\sigma_{h}\right)_{1}\right)$ is

$$
J_{z}\left(\left(\sigma_{h}\right)_{1}\right)=\left(\begin{array}{cc}
1+q\left(z_{1}, z_{2}\right) z_{1} z_{2} & q\left(z_{1}, z_{2}\right) z_{2}^{2} \\
-q\left(z_{1}, z_{2}\right) z_{1}^{2} & 1-q\left(z_{1}, z_{2}\right) z_{1} z_{2}
\end{array}\right)
$$


for some nonzero polynomial $q\left(z_{1}, z_{2}\right) \in K\left[z_{1}, z_{2}\right]$, it is easy to see that this matrix does not belong to $G L_{2}\left(K\left[z_{1}, z_{2}\right]\right)$ because we cannot apply the Euclidean algorithm to its first column.

Example 2.14. A minor modification of the Anick automorphism is the automorphism of $K\langle x, y, z\rangle$ given by

$$
\omega_{m}=\left(x+z(x z-z y)^{m}, y+(x z-z y)^{m} z, z\right) .
$$

Note that the automorphisms $\omega_{m}, m>1$, are not covered by Theorem 2.9, as the polynomials $z(x z-z y)^{m}$ and $(x z-z y)^{m} z$ have no linear components with respect to $x$ and $y$.

Theorem 2.15. The above automorphisms $\omega_{m}$ are wild for all $m \geq 1$.

Proof. Consider the automorphism $\tau=(x+1, y, z)$ of $K\langle x, y, z\rangle$. Clearly, $\omega_{m}$ is wild if and only if $\omega_{m} \tau$ is wild. Direct calculations show that the linear part of the $z$-automorphism

$$
\left.\omega_{m} \tau=x+1+z((x+1) z-z y)^{m}, y+((x+1) z-z y)^{m} z, z\right)
$$

is equal to

$$
\left(\omega_{m} \tau\right)_{1}=\left(x+z \sum_{i=0}^{m-1} z^{i}(x z-z y) z^{m-1-i}, y+\sum_{i=0}^{m-1} z^{i}(x z-z y) z^{m-1-i} z, z\right) .
$$

Hence the matrix $J_{z}\left(\left(\omega_{m} \tau\right)_{1}\right)$ has the form

$$
J_{z}\left(\left(\omega_{m} \tau\right)_{1}\right)=\left(\begin{array}{cc}
1+q\left(z_{1}, z_{2}\right) z_{1} z_{2} & q\left(z_{1}, z_{2}\right) z_{2}^{2} \\
-q\left(z_{1}, z_{2}\right) z_{1}^{2} & 1-q\left(z_{1}, z_{2}\right) z_{1} z_{2}
\end{array}\right)
$$

where $q\left(z_{1}, z_{2}\right)=z_{1}^{m-1}+z_{1}^{m-2} z_{2}+\cdots+z_{2}^{m-1}$. As in Example 2.13 the automorphism $\left(\omega_{m} \tau\right)_{1}$ is wild. Hence $\omega_{m}$ is also wild by Theorem 2.11

It seems plausible that the nontrivial coordinates of $\omega_{m}, m>1$, are wild. However, our methods and the methods in [U2] are not applicable here.

Problem 2. Are the two nontrivial coordinates of the above automorphism $\omega_{m}, m>1$, both wild?

Remark 2.16. The most general form of the result of Umirbaev [U2] shows that the automorphism $\vartheta=(f, g, h)$ of the free metabelian algebra $M(x, y, z)$ is wild if it induces the identity automorphism of $K[x, y, z]$ and the matrix $J_{2}(\vartheta)\left(z_{1}, z_{2}\right)$ cannot be represented as a product of elementary matrices with entries from $K\left[z_{1}, z_{2}\right]$ (see Proposition 2.5). Hence the classes of wild automorphisms and wild coordinates in Theorem 2.9. Example 2.13 and Example 2.14 are not covered by Umirbaev [U2].

Now we are going to show that at least two coordinates of the automorphisms of Umirbaev's class are wild. 
Theorem 2.17. Let $\vartheta=(f, g, h)$ be an automorphism of the free metabelian algebra $M(x, y, z)$ which induces the identity automorphism of $K[x, y, z]$ and the matrix $J_{2}(\vartheta)\left(z_{1}, z_{2}\right)$ does not belong to $G E_{2}\left(K\left[z_{1}, z_{2}\right]\right)$. Then the two coordinates $f(x, y, z)$ and $g(x, y, z)$ are both wild.

Proof. We repeat the main steps of the proof of Theorem 2.6. The polynomial $f(x, y, z)$ $\in M(x, y, z)$ is equal to $x$ modulo the commutator ideal of $M(x, y, z)$ and has the form

$$
f=\sum_{p, q \geq 0} \alpha_{p q} z^{p} x z^{q}+\sum_{p, q \geq 0} \beta_{p q} z^{p} y z^{q}+\sum_{k \geq 2} f_{k}(x, y, z),
$$

where $f_{i}$ is the homogeneous component of degree $i$ in $x, y$ (and $f_{0}=0$ ). Let

$$
a\left(z_{1}, z_{2}\right)=\sum_{p, q \geq 0} \alpha_{p q} z_{1}^{p} z_{2}^{q}, \quad b\left(z_{1}, z_{2}\right)=\sum_{p, q \geq 0} \beta_{p q} z_{1}^{p} z_{2}^{q} .
$$

The polynomials $a\left(z_{1}, z_{2}\right), b\left(z_{1}, z_{2}\right)$ constitute the first column of the matrix $J_{2}(\vartheta)\left(z_{1}, z_{2}\right)$ which does not belong to $G E_{2}\left(K\left[z_{1}, z_{2}\right]\right)$. By Proposition 2.3. $a\left(z_{1}, z_{2}\right), b\left(z_{1}, z_{2}\right)$ cannot be reduced to $(\alpha, 0), 0 \neq \alpha \in K$, by the Euclidean algorithm only.

Now, let $\varphi=(f(x, y, z), u(x, y, z), v(x, y, z))$ be any tame automorphism which sends $x$ to $f(x, y, z)$. Clearly, $\varphi$ induces the tame automorphism

$$
\bar{\varphi}=(\bar{f}, \bar{u}, \bar{v})=(x, \bar{u}, \bar{v})
$$

of the polynomial algebra $K[x, y, z]$. Since $\bar{\varphi}$ fixes $x$, the results in [DY1, DY2, SU1, SU2, SU3] show that $\bar{\varphi}$ is also tame in the class of automorphisms fixing $x$. So, as in the proof of Theorem 2.6. we may replace $\varphi$ with a tame automorphism $\xi=$ $\left(f(x, y, z), u_{1}(x, y, z), v_{1}(x, y, z)\right)$ of $M(x, y, z)$ such that $\xi$ is in the kernel of the natural homomorphism Aut $M(x, y, z) \rightarrow$ Aut $K[x, y, z]$. The tameness of $\xi$ implies that $J_{2}(\xi) \in G E_{2}\left(K\left[z_{1}, z_{2}\right]\right)$. Since the first column of $J_{2}(\xi)$ consists of $a\left(z_{1}, z_{2}\right)$ and $b\left(z_{1}, z_{2}\right)$, this contradicts Proposition 2.5. The considerations for the other coordinate $g$ of $\vartheta$ are similar.

Remark 2.18. Any automorphism $\phi \in$ Aut $K\langle x, y, z\rangle$ which induces an automorphism in Aut $M(x, y, z)$ of the type in Theorem 2.17 (in other words, any automorphism in Aut $K\langle x, y, z\rangle$ obtained by lifting an automorphism in Aut $M(x, y, z)$ of the type in Theorem 2.17) is a wild automorphism containing at least two wild coordinates.

The above results suggest the following problems.

Problem 3. Is it true that the two nontrivial coordinates of a wild automorphism of $K\langle x, y, z\rangle$ fixing $z$ are both wild?

Problem 4. Is it true that every wild automorphism of $K\langle x, y, z\rangle$ contains at least two wild coordinates? 


\section{Special wild automorphisms of the free metabelian algebra}

In this section we shall construct a wild automorphism $\tau$ of the free metabelian algebra $M(x, y, z)$ over any field $K$ of arbitrary characteristic with the following properties:

(i) $\tau=(f(x, y, z), y, z)$ fixes two of the variables. (Hence Lemma 2.1] does not hold for $M(x, y, z)$.)

(ii) The Jacobian matrix $J_{M}(\tau)$ is a product of elementary matrices.

(iii) $\tau$ cannot be lifted to an automorphism of $K\langle x, y, z\rangle$.

Recall the definition of the Fox derivatives of the free algebra $K\langle X\rangle$ (see e.g. [MSY]). If

$$
f(X)=\sum_{i=1}^{n} x_{i} f_{i}(X)+\alpha, \quad \alpha \in K, f_{i}(X) \in K\langle X\rangle,
$$

then the right Fox derivatives of $f(X)$ are

$$
\frac{\partial_{r} f}{\partial_{r} x_{i}}=f_{i}(X), \quad i=1, \ldots, n
$$

Similarly, if

$$
f(X)=\sum_{i=1}^{n} f_{i}(X) x_{i}+\alpha, \quad \alpha \in K, f_{i}(X) \in K\langle X\rangle,
$$

then the left Fox derivatives of $f(X)$ are

$$
\frac{\partial_{l} f}{\partial_{l} x_{i}}=f_{i}(X), \quad i=1, \ldots, n .
$$

The right and left Jacobian matrices of an endomorphism $\varphi$ of $K\langle X\rangle$ are, respectively,

$$
J_{r}(\varphi)=\left(\frac{\partial_{r} \varphi\left(x_{j}\right)}{\partial_{r} x_{i}}\right), \quad J_{l}(\varphi)=\left(\frac{\partial_{l} \varphi\left(x_{j}\right)}{\partial_{l} x_{i}}\right) .
$$

The chain rule implies that if $\varphi$ is an automorphism, then $J_{r}(\varphi)$ and $J_{l}(\varphi)$ are invertible (but the opposite is not true in the general case).

We need some machinery from $[\overline{\mathrm{BGLM}}]$ and $[\overline{\mathrm{BD}}]$. We describe it in the case of three variables only. We define an equivalence relation $\sim$ on $K\langle x, y, z\rangle$. We say that two monomials $u$ and $v$ are equivalent if they can be obtained from each other by cyclic permutation (i.e., $u \sim v$ if and only if $u=w_{1} w_{2}$ and $v=w_{2} w_{1}$ for some monomials $\left.w_{1}, w_{2}\right)$, and then extend $\sim$ to $K\langle x, y, z\rangle$ by linearity.

Proposition 3.1 ([BGLM] $\mathrm{BD}]$ ). Let $\sigma$ be an endomorphism of $K\langle x, y, z\rangle$ which is equal to the identity of $K\langle x, y, z\rangle$ modulo the $k$-th degree of the augmentation ideal, i.e.

$$
\sigma=\left(x+f_{k}+\cdots+f_{m}, y+g_{k}+\cdots+g_{m}, z+h_{k}+\cdots+h_{m}\right),
$$

where $f_{i}, g_{i}, h_{i}$ are the homogeneous components of degree $i$ of $\sigma(x), \sigma(y), \sigma(z)$, respectively. If $\sigma$ is an automorphism and $k \geq 2$, then the homogeneous component of degree 
$k-1$ of the trace of the right Jacobian matrix

$$
\frac{\partial_{r} f_{k}}{\partial_{r} x}+\frac{\partial_{r} g_{k}}{\partial_{r} y}+\frac{\partial_{r} h_{k}}{\partial_{r} z}
$$

is equivalent to 0 . A similar statement holds for the trace of the left Jacobian matrix.

Theorem 3.2. The endomorphism

$$
\tau=\left(x+x^{2}[y, z], y, z\right)
$$

of the free metabelian algebra $M(x, y, z)$ is a wild automorphism which cannot be lifted to an automorphism of $K\langle x, y, z\rangle$. Its Jacobian matrix

$$
J_{M}(\tau)=\left(\begin{array}{ccc}
1 & 0 & 0 \\
x_{1}^{2}\left(z_{2}-z_{1}\right) & 1 & 0 \\
x_{1}^{2}\left(y_{1}-y_{2}\right) & 0 & 1
\end{array}\right)
$$

is a product of two elementary matrices.

Proof. Obviously $\tau$ is an automorphism and $\tau^{-1}=\left(x-x^{2}[y, z], y, z\right)$. Also, its Jacobian matrix $J_{M}(\tau)$ is a product of elementary matrices. Now, suppose $\tau$ lifts to an automorphism $\sigma$ of $K\langle x, y, z\rangle$. Then

$$
\sigma=\left(x+x^{2}[y, z]+f(x, y, z), y+g(x, y, z), z+h(x, y, z)\right),
$$

where $f(x, y, z), g(x, y, z), h(x, y, z)$ belong to the T-ideal generated by the polynomial identity $\left[x_{1}, x_{2}\right]\left[x_{3}, x_{4}\right]=0$. Hence $f, g, h$ have no homogeneous components of degree $\leq 3$ and

$$
f=f_{4}+\cdots+f_{m}, \quad g=g_{4}+\cdots+g_{m}, \quad h=h_{4}+\cdots+h_{m},
$$

where $f_{i}, g_{i}, h_{i}$ are homogeneous of degree $i$. Clearly, the components $f_{4}, g_{4}, h_{4}$ are linear combinations of products of two commutators of the variables. By Proposition 3.1 .

$$
\begin{gathered}
\frac{\partial_{r}\left(x^{2}[y, z]+f_{4}\right)}{\partial_{r} x}+\frac{\partial_{r} g_{4}}{\partial_{r} y}+\frac{\partial_{r} h_{4}}{\partial_{r} z} \sim 0, \\
\frac{\partial_{l}\left(x^{2}[y, z]+f_{4}\right)}{\partial_{l} x}+\frac{\partial_{l} g_{4}}{\partial_{l} y}+\frac{\partial_{l} h_{4}}{\partial_{l} z} \sim 0 .
\end{gathered}
$$

Since $x^{2}[y, z]=x^{2} y z-x^{2} z y$, we obtain

$$
\frac{\partial_{r} x^{2}[y, z]}{\partial_{r} x}=x[y, z] \sim x y z-x z y, \quad \frac{\partial_{l} x^{2}[y, z]}{\partial_{l} x}=0 .
$$

The components of (3) and (4) which are multilinear in $x, y, z$ are equivalent to 0 . The components of the Fox derivatives

$$
\frac{\partial_{r} f_{4}}{\partial_{r} x}, \quad \frac{\partial_{r} g_{4}}{\partial_{r} y}, \quad \frac{\partial_{r} h_{4}}{\partial_{r} z}
$$


which are multilinear in $x, y, z$ come, respectively, from

$$
\begin{aligned}
& f_{4}^{\prime}=\alpha_{1}[x, y][x, z]+\beta_{1}[x, z][x, y], \\
& g_{4}^{\prime}=\alpha_{2}[x, y][y, z]+\beta_{2}[y, z][x, y], \\
& h_{4}^{\prime}=\alpha_{3}[x, z][y, z]+\beta_{3}[y, z][x, z] .
\end{aligned}
$$

Direct calculations give

$$
\begin{aligned}
\frac{\partial_{r} f_{4}^{\prime}}{\partial_{r} x}+ & \frac{\partial_{r} g_{4}^{\prime}}{\partial_{r} y}+\frac{\partial_{r} h_{4}^{\prime}}{\partial_{r} z} \\
& \sim\left(\alpha_{1} y[x, z]+\beta_{1} z[x, y]\right)+\left(-\alpha_{2} x[y, z]+\beta_{2} z[x, y]\right)-\left(\alpha_{3} x[y, z]+\beta_{3} y[x, z]\right) \\
& \sim\left(-\alpha_{1}+\beta_{1}-\alpha_{2}+\beta_{2}-\alpha_{3}+\beta_{3}\right)(x y z-x z y) .
\end{aligned}
$$

Together with (5) this implies that

$$
-\alpha_{1}+\beta_{1}-\alpha_{2}+\beta_{2}-\alpha_{3}+\beta_{3}+1=0 .
$$

Similarly,

$$
\begin{aligned}
\frac{\partial_{l} f_{4}^{\prime}}{\partial_{l} x} & +\frac{\partial_{l} g_{4}^{\prime}}{\partial_{l} y}+\frac{\partial_{l} h_{4}^{\prime}}{\partial_{l} z} \\
& \sim-\left(\alpha_{1}[x, y] z+\beta_{1}[x, z] y\right)+\left(-\alpha_{2}[x, y] z+\beta_{2}[y, z] x\right)+\left(\alpha_{3}[x, z] y+\beta_{3}[y, z] x\right) \\
& \sim\left(-\alpha_{1}+\beta_{1}-\alpha_{2}+\beta_{2}-\alpha_{3}+\beta_{3}\right)(x y z-x z y) \sim 0
\end{aligned}
$$

in virtue of (5). Hence

$$
-\alpha_{1}+\beta_{1}-\alpha_{2}+\beta_{2}-\alpha_{3}+\beta_{3}=0 .
$$

Clearly, (6) and (7) contradict each other. Hence $\tau$ cannot be lifted to an automorphism of $K\langle x, y, z\rangle$ and, therefore, is a wild automorphism of $M(x, y, z)$.

Problem 5. Is the polynomial $x+x^{2}[y, z]$ a wild coordinate of $M(x, y, z)$ ? Can it be lifted to a coordinate of $K\langle x, y, z\rangle$ ?

Problem 6. Do there exist wild automorphisms and wild coordinates of the free metabelian algebra $M(X)$ of rank $n>3$ ? Are there wild automorphisms similar to the automorphism $\tau$ constructed above?

\section{Lifting of automorphisms fixing variables}

The considerations in this section work over an arbitrary field of any characteristic.

Let $G(X)$ be the free group generated by the finite set $X$. The theorem of Nielsen [Ni] states that every automorphism of $G(X)$ is a product of the elementary automorphisms $\left(x_{1}^{-1}, x_{2}, \ldots, x_{n}\right),\left(x_{1} x_{2}, x_{2}, \ldots, x_{n}\right)$, and $\left(x_{\sigma(1)}, \ldots, x_{\sigma(n)}\right)$, where $\sigma$ belongs to the symmetric group $S_{n}$. The proof of Nielsen gives also an algorithm which finds such 
a decomposition. The theorem of Schreier [Sch] states that every subgroup of the free group with any number of generators is also free.

There are several important varieties of algebras over a field with free objects which share the above properties of free groups. A variety $\mathfrak{V}$ of algebras is called Schreier if the subalgebras of the relatively free algebras $F(\mathfrak{V})$ are again relatively free, where $F(\mathfrak{V})$ is freely generated by a set of any cardinality. The variety $\mathfrak{V}$ is Nielsen if all automorphisms of the free algebras $F_{n}(\mathfrak{V})$ of finite rank are tame. A theorem of Lewin $[\mathrm{L}]$ implies that over an infinite field $K$ the two notions coincide, i.e., $\mathfrak{V}$ is Nielsen if and only if it is Schreier. The same holds over an arbitrary field $K$, provided that the variety $\mathfrak{V}$ is defined by a multilinear system of polynomial identities. See the book [MSY] for more details about examples of Schreier varieties, and the properties of the subalgebras and the automorphisms of their free objects.

The variety of all (not necessarily associative) algebras is Schreier, by the theorem of Kurosh [Ku]. Recall that the absolutely free algebra $K\{X\}$ consists of all polynomials in the set of noncommuting and nonassociative variables $X$, e.g. $(x x) x \neq x(x x)$. One of the key moments of the proof of Kurosh (and of all other proofs that some varieties are Schreier) is the following (see [MSY, Theorem 11.1.1]). For a nonzero polynomial $f \in K\{X\}$ we denote by $\bar{f}$ the homogeneous component of maximal degree of $f$.

Proposition 4.1. (i) Any finite set $S$ of $K\{X\}$ can be transformed into a set of free generators of the subalgebra generated by $S$ by a finite sequence of elementary transformations (with cancellation of possible zeros).

(ii) If $F=\left\{f_{1}, \ldots, f_{n}\right\}$ is a set of free generators of $K\{X\}$, and $g \in K\{X\}$, then $\bar{g}$ belongs to the subalgebra of $K\{X\}$ generated by $\bar{f}_{1}, \ldots, \bar{f}_{n}$.

For an automorphism $\varphi=\left(f_{1}, \ldots, f_{n}\right)$ of $K\{X\}$ we define the degree of $\varphi$ as the sum of the degrees of the coordinates $f_{i}$ :

$$
\operatorname{deg}(\varphi)=\sum_{i=1}^{n} \operatorname{deg}\left(f_{i}\right)
$$

Clearly, $\operatorname{deg}(\varphi) \geq n$. The following consequence of Proposition 4.1 can be used effectively to decompose an automorphism of $K\{X\}$ as a product of elementary automorphisms.

Corollary 4.2. Let $\varphi=\left(f_{1}, \ldots, f_{n}\right) \in$ Aut $K\{X\}$ with $\operatorname{deg}(\varphi)>n$. Then there exists an integer $i$ and a polynomial $g\left(y_{1}, \ldots, y_{i-1}, y_{i+1}, \ldots, y_{n}\right)$ such that

$$
\bar{f}_{i}=g\left(\bar{f}_{1}, \ldots, \bar{f}_{i-1}, \bar{f}_{i+1}, \ldots, \bar{f}_{n}\right) .
$$

Let $\tau$ be the elementary automorphism of $K\{X\}$ defined by

$$
\tau=\left(x_{1}, \ldots, x_{i-1}, x_{i}-g\left(x_{1}, \ldots, x_{i-1}, x_{i+1}, \ldots, x_{n}\right), x_{i+1}, \ldots, x_{n}\right) .
$$

Then $\operatorname{deg}(\varphi \tau)<\operatorname{deg}(\varphi)$.

Now we are able to prove the following. 
Theorem 4.3. Let

$$
\varphi=\left(f_{1}(X, Z), \ldots, f_{n}(X, Z), z_{1}, \ldots, z_{m}\right) \in \operatorname{Aut}_{Z} K\{X, Z\}
$$

be an automorphism of $K\{X, Z\}$ fixing the variables $Z$. Then $\varphi$ is tame in the class of Z-automorphisms.

Proof. Let us consider $\varphi$ as an automorphism of $K\{X, Z\}$ in the usual sense. The total degree of $\varphi$ is

$$
\operatorname{deg}(\varphi)=\sum_{i=1}^{n} \operatorname{deg}\left(f_{i}(X, Z)\right)+\sum_{j=1}^{m} \operatorname{deg}\left(z_{j}\right)=\sum_{i=1}^{n} \operatorname{deg}\left(f_{i}\right)+m
$$

Since $\varphi$ is a $Z$-automorphism, each polynomial $f_{1}, \ldots, f_{n}$ essentially depends on $X$.

If $\operatorname{deg}(\varphi)=n+m$, then all polynomials $f_{i}(X, Z)$ are of total degree 1 and $\varphi$ is affine. We replace $\varphi$ with the product $\psi=\varphi \tau_{0}$, where $\tau_{0}$ is the translation

$$
\tau_{0}=\left(x_{1}-f_{1}(0,0), \ldots, x_{n}-f_{n}(0,0), z_{1}, \ldots, z_{m}\right) .
$$

Clearly, $\tau_{0}$ is a product of $Z$-elementary automorphisms and $\psi$ is a linear automorphism. Its matrix, as a linear operator of the vector space with basis $X \cup Z$, is

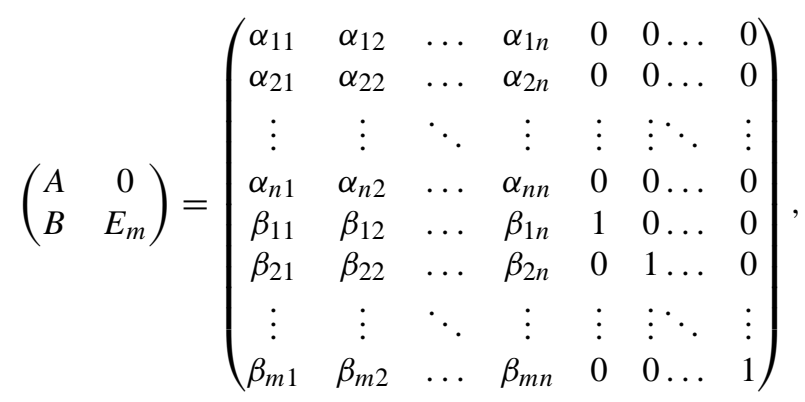

and $A=\left(\alpha_{p q}\right), B=\left(\beta_{r s}\right)$ are, respectively, $n \times n$ and $m \times n$ matrices with entries in $K$, $E_{m}$ is the $m \times m$ identity matrix, and $A$ is invertible. Since we work over a field, $A$ is a product of elementary matrices and this implies that, multiplying $\psi$ by a product of elementary linear automorphisms fixing $Z$, we bring it to the automorphism

$$
\tau_{1}=\left(x_{1}+g_{1}(Z), \ldots, x_{n}+g_{n}(Z), z_{1}, \ldots, z_{m}\right),
$$

which is a product of elementary automorphisms fixing $Z$.

Now, let $\operatorname{deg}(\varphi)>n+m$. Then at least one of the polynomials $f_{i}(X, Z)$ is not linear. The leading components of the $n+m$ coordinates are

$$
\overline{f_{1}(X, Z)}, \ldots, \overline{f_{n}(X, Z)}, \quad \bar{z}_{1}=z_{1}, \ldots, \bar{z}_{m}=z_{m} .
$$

By Corollary 4.2 one of these homogeneous components can be expressed by the others. Obviously, $z_{j}$ cannot be expressed as a polynomial of $\bar{f}_{1}, \ldots, \bar{f}_{n}$ and the other $z_{1}, \ldots, z_{j-1}, z_{j+1}, \ldots, z_{m}$. It follows that some $\bar{f}_{i}$ is a polynomial of $\bar{f}_{1}, \ldots, \bar{f}_{i-1}$, 
$\bar{f}_{i+1}, \ldots, \bar{f}_{n}$ and $z_{1}, \ldots, z_{m}$. This implies that the elementary automorphism $\tau$ of $K\{X, Z\}$ given by Corollary 4.2 is of the form

$$
\tau=\left(x_{1}, \ldots, x_{i-1}, x_{i}-g(X, Z), x_{i+1}, \ldots, x_{n}, z_{1}, \ldots, z_{m}\right),
$$

where $g(X, Z)$ does not depend on $x_{i}$. Then $\operatorname{deg}(\varphi \tau)<\operatorname{deg}(\varphi)$ and the proof is completed by an obvious induction on the degree of $\varphi$.

The theorem below is an immediate concequence of Theorem 4.3 .

Theorem 4.4. Let $\varphi$ be an automorphism of $K[X, Z]$ or $K\langle X, Z\rangle$ which fixes $Z$. If $\varphi$ is wild as a $Z$-automorphism, then it cannot be lifted to an automorphism of $K\{X, Z\}$ which also fixes $Z$.

Remark 4.5. The Nagata automorphism is wild as a $z$-automorphism of $K[x, y, z]$ (see $[\mathrm{N}]$ ), as well as wild in the usual sense [SU1, [SU3]. Hence it cannot be lifted to any automorphism of $K\{x, y, z\}$. On the other hand, by the theorem of Smith [Sm], automorphisms of $K[X]$ of a large class become tame as automorphisms of $K[X, t]$ if we extend them to act identically on $t$. In particular, the extension of the Nagata automorphism

$$
v^{\prime}=\left(x-2 y\left(y^{2}+x z\right)-z\left(y^{2}+x z\right)^{2}, y+z\left(y^{2}+x z\right), z, t\right)
$$

is tame as an automorphism of $K[x, y, z, t]$. It is easy to see that it is wild in the class of automorphisms of $K[x, y, z, t]$ fixing $z$ and $t$. Hence, Theorem 4.4 shows that $v^{\prime}$ cannot be lifted to an automorphism of $K\{x, y, z, t\}$ which fixes $z$ and $t$.

Similarly, the automorphism of Anick is wild as an automorphism fixing a variable [DY3] and even wild in the usual sense [U2]. But it becomes tame when extended to an automorphism of $K\langle x, y, z, t\rangle$. The technique of [DY3] implies that the extension of the Anick automorphism

$$
(x+z(x z-z y), y+(x z-z y) z, z, t)
$$

is wild in the group of automorphisms of $K\langle x, y, z, t\rangle$ which fix $z, t$, although this automorphism is tame in the usual sense. Hence, our theorem shows that it cannot be lifted to an automorphism of $K\{x, y, z, t\}$ which fixes $z, t$.

We conclude this section with several open problems.

Problem 7. (i) If $\varphi$ is an automorphism of $K[X]$, can it be lifted to an automorphism of $K\langle X\rangle$ ? (If $\varphi$ is wild, and nevertheless the answer is positive, this would mean that it is not "too wild".)

(ii) If $\varphi \in \operatorname{Aut}_{Z} K[X, Z]$, can it be lifted to a $Z$-automorphism of $K\langle X, Z\rangle$ ? Are Z-wild automorphisms wild also in the usual sense?

Problem 8. How far can one lift the automorphisms of $K[X]$ ? Describe the varieties $\mathfrak{V}$ of algebras with the property that every automorphism of $K[X]$ can be lifted to an automorphism of the relatively free algebra $F_{n}(\mathfrak{V})$ of rank $n=|X|$. 
For example, a theorem of Umirbaev [U1] implies that every automorphism of $K[X]$ can be lifted to an automorphism of the free metabelian algebra $M(X)$.

Problem 9. (i) If $p(X)$ is a coordinate of $K[X]$, can it be lifted to a coordinate of $K\langle X\rangle$ ?

(ii) How far can one lift the coordinates of $K[X]$ ? Describe the varieties $\mathfrak{V}$ of algebras with the property that every coordinate of $K[X]$ can be lifted to a coordinate of $F_{n}(\mathfrak{V})$.

(iii) Can the two nontrivial Nagata coordinates $x-2 y\left(y^{2}+x z\right)-z\left(y^{2}+x z\right)^{2}$ and $y+z\left(y^{2}+x z\right)$ be lifted to coordinates of $K\langle x, y, z\rangle$ ?

Acknowledgments. The authors are grateful to Leonid Makar-Limanov, Ivan Shestakov and Efim Zelmanov for insightful discussion.

The research of Vesselin Drensky was partially supported by Grant MM-1106/2001 of the Bulgarian National Science Fund.

The research of Jie-Tai Yu was partially supported by a Hong Kong RGC-CERG Grant.

\section{References}

[BD] Bryant, R. M., Drensky, V.: Obstructions to lifting automorphisms of free algebras. Comm. Algebra 21, 4361-4389 (1993) Zbl 0797.16032 MR 1242836

[BGLM] Bryant, R. M., Gupta, C. K., Levin, F., Mochizuki, H. Y.: Nontame automorphisms of free nilpotent groups. Comm. Algebra 18,3619-3631 (1990) Zbl 0722.20017 MR 1068609

[C1] Cohn, P. M.: On the structure of the $\mathrm{GL}_{2}$ of a ring. Inst. Hautes Études Sci. Publ. Math. 30, 5-53 (1966) Zbl 0144.26301 MR 0207856

[C2] Cohn, P. M.: Free Rings and Their Relations. 2nd ed., Academic Press (1985) Zbl 0659.16001 MR 0800091

[Cz] Czerniakiewicz, A. J.: Automorphisms of a free associative algebra of rank 2. I, II. Trans. Amer. Math. Soc. 160, 393-401 (1971); 171, 309-315 (1972) Zbl 0227.16001. 0258.16001 MR 0280549, 0310021

[DL] Dicks, W., Lewin, J.: A Jacobian conjecture for free associative algebras. Comm. Algebra 10, 1285-1306 (1982) Zbl 0493.16005 MR 0660345

[DGY] Drensky, V., Gutierrez, J., Yu, J.-T.: Gröbner bases and the Nagata automorphism. J. Pure Appl. Algebra 135, 135-153 (1999) Zbl 0932.13013 MR 1667554

[DY1] Drensky, V., Yu, J.-T.: Automorphisms and coordinates of polynomial algebras. In: Combinatorial and Computational Algebra (Hong Kong, 1999), K.Y. Chan et al. (eds.), Contemp. Math. 264, Amer. Math. Soc., 179-206 (2000) Zbl 0998.14026 MR 1800695

[DY2] Drensky, V., Yu, J.-T.: Tame and wild coordinates of $K[z][x, y]$. Trans. Amer. Math. Soc. 353, 519-537 (2001) Zbl 0965.13008 MR 1709773

[DY3] Drensky, V., Yu, J.-T.: Automorphisms fixing a variable of $K\langle x, y, z\rangle$. J. Algebra 291, 250-258 (2005) Zbl 1086.16019 MR 2158521

[J] Jung, H. W. E.: Über ganze birationale Transformationen der Ebene. J. Reine Angew. Math. 184, 161-174 (1942) Zbl 0027.08503 MR 0008915

[K] van der Kulk, W.: On polynomial rings in two variables. Nieuw Arch. Wisk. (3) 1, 33-41 (1953) Zbl 0050.26002 MR 0054574

[Ku] Kurosh, A.: Nonassociative free algebras and free products of algebras. Mat. Sb. 20, 239-262 (1947) (in Russian) Zbl 0041.16803 MR 0020986 
[L] Lewin, J.: On Schreier varieties of linear algebras. Trans. Amer. Math. Soc. 132, 553-562 (1968) Zbl 0172.04201 MR 0224663

[ML1] Makar-Limanov, L. G.: On automorphisms of free algebra with two generators. Funktsional. Anal. i Prilozhen. 4, no. 3, 107-108 (1970) (in Russian); English transl.: Funct. Anal. Appl. 4, 262-263 (1970) Zbl 0218.13006 MR 0271161

[ML2] Makar-Limanov, L. G.: On automorphisms of certain algebras. Ph.D. Thesis, Moscow (1970) (in Russian)

[MSY] Mikhalev, A. A., Shpilrain, V., Yu, J.-T.: Combinatorial Methods. Free Groups, Polynomials, Free Algebras. CMS Books in Math., Springer, New York (2004) Zbl 1039.16024 MR 2014326

[N] Nagata, M.: On Automorphism Group of $k[x, y]$. Lect. in Math. 5, Kyoto Univ., Kinokuniya, Tokyo (1972) Zbl 0306.14001 MR 0337962

[Ni] Nielsen, J.: Über die Isomorphismen unendlicher Gruppen ohne Relation. Math. Ann. 79, 269-272 (1918) JFM 46.0175.02 MR 1511927

[P1] Park, H.: A computational theory of Laurent polynomial rings and multidimensional FIR systems. Ph.D. Thesis, Univ. of California, Berkeley (1995)

[P2] Park, H.: A realization algorithm for $\operatorname{SL}\left(R\left[x_{1}, \ldots, x_{m}\right]\right)$ over the Euclidean domain. SIAM J. Matrix Anal. Appl. 21, 178-184 (1999) Zbl 0953.15027 MR 1709733

[Sc] Schofield, A. N.: Representation of Rings over Skew Fields. London Math. Soc. Lecture Note Ser. 92, Cambridge Univ. Press, Cambridge (1985) Zbl 0571.16001 MR 0800853

[Sch] Schreier, O.: Die Untergruppen der freien Gruppen. Abh. Math. Sem. Univ. Hamburg 5, 161-183 (1927) JFM 53.0110.01

[SU1] Shestakov, I. P., Umirbaev, U. U.: The Nagata automorphism is wild. Proc. Nat. Acad. Sci. U.S.A. 100, 12561-12563 (2003) Zbl 1065.13010 MR 2017754

[SU2] Shestakov, I. P., Umirbaev, U. U.: Poisson brackets and two-generated subalgebras of rings of polynomials. J. Amer. Math. Soc. 17, 181-196 (2004) Zbl 1044.17014 MR 2015333

[SU3] Shestakov, I. P., Umirbaev, U. U.: The tame and the wild automorphisms of polynomial rings in three variables. J. Amer. Math. Soc. 17, 197-227 (2004) Zbl 1056.14085 MR 2015334

[SY] Shpilrain, V., Yu, J.-T.: Polynomial automorphisms and Gröbner reductions. J. Algebra 197, 546-558 (1997) Zbl 0899.13029 MR 1483780

[Sm] Smith, M. K.: Stably tame automorphisms. J. Pure Appl. Algebra 58, 209-212 (1989) Zbl 0692.13004 MR 1001475

[THK] Tolhuizen, L., Hollmann, H., Kalker, T. A. C. M.: On the realizability of biorthogonal, $m$-dimensional two-band filter banks. IEEE Trans. Signal Proces. 43, 640-648 (1995)

[U1] Umirbaev, U. U.: On the extension of automorphisms of polynomial rings. Sibirsk. Mat. Zh. 36, 911-916 (1995) (in Russian); English transl.: Siberian Math. J. 36, 787-791 (1995) Zbl 0866.16023 MR 1367258

[U2] Umirbaev, U. U.: Tame and wild automorphisms of polynomial algebras and free associative algebras. J. Algebra, to appear

[UY] Umirbaev, U. U., Yu, J.-T.: The strong Nagata conjecture. Proc. Nat. Acad. Sci. U.S.A. 101, 4352-4355 (2004) Zbl 1065.13011 MR 2055075 